\title{
The efficacy of sustained-release chitosan microspheres containing recombinant human parathyroid hormone on MRONJ
}

\author{
Aysegul ERTEN TAYSI(a) \\ Erdal CEVHER ${ }^{(b)}$ \\ Melike SESSEVMEZ(b) iD \\ Vakur OLGAC(c) \\ Nuri MERT TAYSI(d) (D) \\ Berkem ATALAY(e) \\ (a)Altinbas University, Faculty of Dentistry, \\ Department of Oral and Maxillofacial \\ Surgery, Istanbul, Turkey. \\ (b)|stanbul University, Faculty of Pharmacy, \\ Department of Pharmaceutical Technology, \\ Istanbul, Turkey. \\ (c)| Istanbul University, Institute of Oncology, \\ Department of Tumor Pathology and \\ Cytology, Istanbul, Turkey. \\ (d) Istinye University, Vocational School of \\ Health Care Services, Istanbul, Turkey. \\ (e) Istanbul University-Cerrahpaşa, Vocational \\ School of Health Care Services, \\ Istanbul, Turkey. \\ Declaration of Interests: The authors \\ certify that they have no commercial or \\ associative interest that represents a conflict \\ of interest in connection with the manuscript.
}

Corresponding Author:

Berkem Atalay

E-mail: berkematalay@gmail.com

Submitted: March 23, 2019

Accepted for publication: July 23, 2019

Last revision: August 6, 2019

\begin{abstract}
Treatment of patients with bisphosphonate usage is a significant concern for oral surgeons because it interferes with jaw bone turnover and regeneration. In case of adverse effects manifesting related to bisphosphonate use, oral surgeons are usually treating and keep the patient's symptoms under control. In this study, we aimed to investigate a new treatment protocol for medication-related osteonecrosis of the jaw (MRONJ). This treatment protocol consisted of administering human parathyroid hormone (hPTH) loaded chitosan microspheres which were prepared by ionotropic gelation method or/and the prepared microspheres were suspended in a poloxamer gel. After in-vitro optimization studies, the efficacy of the chosen formulations was evaluated in-vivo studies. Zoledronic acid was administered daily to forty-eight adult female Sprague-Dawley rats, divided into four experimental groups, at a daily concentration of $0.11 \mathrm{mg} / \mathrm{kg}$ over three weeks to induce the MRONJ model. At the end of this period, maxillary left molar teeth were extracted. In the first group, the subjects received no treatment. In the negative control group, poloxamer hydrogel containing empty microspheres were immediately applied to the soft tissues surrounding the extraction socket. The treatment group-1 was treated with local injections of poloxamer hydrogel containing hPTH. The treatment group-2 was treated with a single local injection of poloxamer hydrogel containing hPTH-loaded chitosan microspheres. Both treatment groups received a total of $7 \mu \mathrm{g}$ of hPTH at the end of the treatment protocol. Our study demonstrates successful attenuation of MRONJ through a local drug delivery system combined with $\mathrm{hPTH}$, as opposed to previously attempted treatment strategies.
\end{abstract}

Keywords: Osteonecrosis; Teriparatide; Chitosan; Microspheres.

\section{Introduction}

Synthetic, chemically stable, and nonhydrolyzable analogues of inorganic pyrophosphates known as bisphosphonates are antiresorptive medications prescribed to manage bone pathologies that are characterized by excessive bone resorption such as osteoporosis, Paget's disease, and 
cancer-related conditions. ${ }^{1,2}$ Bisphosphonates are generally potent inhibitors of bone resorption that selectively affect osteoclast formation in vivo. ${ }^{3}$

There is a variety of bisphosphonates that may be prescribed according to their various biological and clinical potencies. ${ }^{4,5}$ While the effects of a variety of bisphosphonates on the bone metabolism has been extensively investigated, the pathogenesis of their adverse effects on craniofacial bones has not been fully elucidated to date. ${ }^{6,78}$

Medication-related osteonecrosis of jaw (MRONJ) has emerged as a serious condition in patients undergoing long-term treatment with high doses of bisphosphonates, mainly after tooth extraction. ${ }^{9}$ The pathogenesis of MRONJ is still not fully elucidated, but it is known that there is an elevated risk from intravenous administration compared to oral use. ${ }^{7}$ In addition, several pathogenic factors, including osteoclast inhibition, antiangiogenic processes, soft tissue toxicity and immune dysfunction play a vital role in MRONJ.7 Furthermore, the success rate of treatment is low even with surgical intervention. While the current consensus on managing MRONJ is to eliminate pain, control infection of the soft and hard tissues, and minimize the progression or occurrence of bone necrosis, the treatment of patients with MRONJ remains a challenge and an ideal standard procedure has not yet been established. ${ }^{7,8}$ For these reasons, the application of alternative treatments such as platelet-rich plasma, ${ }^{10}$ low-level laser irradiation, ${ }^{11}$ bone morphogenic protein, ${ }^{12}$ and human parathyroid hormone $(\mathrm{hPTH})^{13}$ have been suggested.

Endogenous hPTH is an 84 -amino acid peptide (PTH 1-84) produced in the parathyroid glands. Additionally, the partial peptide PTH 1-34, known as teriparatide, is an active recombinant form of PTH. $\mathrm{hPTH}$ can have anabolic or catabolic effects on bone tissues, depending on the length of exposure and dosage regimen. Although intermittent exposure to $\mathrm{hPTH}$ leads to an anabolic effect that increases osteoblastic bone formation, continuous exposure leads to a catabolic effect that stimulates bone resorption..$^{14}$ In several studies, systemic intermittent administration of teriparatide caused significant improvements in bone healing, but no topical application of teriparatide on the bone has been attempted to treat MRONJ. ${ }^{15,16,17}$
Chitosan, a natural linear polyaminosaccharide, is obtained by the alkaline deacetylation of chitin. ${ }^{18}$ Favorable properties of chitosan such as biodegradability, low toxicity and good biocompatibility make it suitable for use in biomedical and pharmaceutical applications. ${ }^{19,20}$ Ionic gelation is one of the most common techniques for the preparation of micro- or nanoparticles for the sustained release of macromolecules such as peptides and proteins owing to its simple and mild manufacturing process that minimizes the aggregation and denaturation of active molecules during the particle fabrication process. The cationic structure of chitosan allows the formation of ionic complexes with multivalent anions such as tripolyphosphate (TPP), which extend the duration of drug release..$^{21}$

Poloxamers, which are nonionic block copolymers composed of a central hydrophobic chain (polyoxypropylene) bordered by two hydrophilic chains of polyoxyethylene, can form thermoreversible gels-depending on concentration and temperature-due to their amphiphilic structure, and increase drug residence time in an application site, resulting in enhanced efficacy. ${ }^{22}$ Poloxamer 127 is frequently used to prepare thermoreversible gel formulations due to its biodegradability, low toxicity, good drug release characteristics and capability to transform from a viscous liquid to a transparent gel at body temperature. ${ }^{23}$

In this study, the role of sustained-release thermoreversible poloxamer gel formulations containing hPTH or hPTH-loaded chitosan microspheres on MRONJ in an experimental animal model was investigated by histomorphometrical assessments. This was done to identify novel sustained-release local drug delivery systems for increased treatment effectiveness.

\section{Methodology}

\section{In vitro studies}

\section{Preparation of hPTH-loaded chitosan microspheres \\ $\mathrm{hPTH}$, which was used to prepare hPTH-loaded chitosan microspheres, was isolated from the}


commercial product, Forsteo, by dialysis. Dialysis was carried out against distilled water in a 2-L volumetric flask in a dark room using a cellulose dialysis membrane (Visking dialysis tubing cutoff: 3,500 Da; Medicell, London, UK). The purified PTH solution was freeze-dried (Virtis Advantage, New York, USA) at $-45^{\circ} \mathrm{C}$ for $24 \mathrm{~h}$, and the structural integrity of powdered hPTH was confirmed by SDS-PAGE (data not shown). hPTH-loaded chitosan microspheres were fabricated by ionotropic gelation using TPP as the cross-linker. ${ }^{24}$ Next, $0.6 \mathrm{~mL}$ TPP solution in water at concentrations between $0.125 \%$ and $0.25 \%(\mathrm{w} / \mathrm{v})$ was added dropwise to $1.4 \mathrm{~mL}$ chitosan solution in $1 \%$ acetic acid at concentrations between $0.25 \%$ and $0.5 \%(\mathrm{w} / \mathrm{v})$ containing $500 \mu \mathrm{g}$ $\mathrm{hPTH}$, followed by incubation with stirring in a magnetic stirrer at $600 \mathrm{rpm}$ for $3 \mathrm{~h}$. The microspheres were collected by centrifugation (Optima XPN Ultracentrifuge, Beckman, Indianapolis, USA) at $30,000 \mathrm{rpm}$, freeze-dried, and then sterilized by $\gamma$-irradiation (15 kGy) using Cobalt-60 $\left({ }^{60} \mathrm{Co}\right)$.

\section{Characterization of hPTH-loaded chitosan microspheres}

The microsphere production yield was calculated by gravimeter. A total of $20 \mathrm{~mL}$ microsphere dispersion was centrifuged at $50,000 \mathrm{rpm}$ for $2 \mathrm{~h}$ at $10^{\circ} \mathrm{C}$, and then the particles were lyophilized at $-40^{\circ} \mathrm{C}$ for $24 \mathrm{~h}$. The production yield was calculated as follows:

Production yield $(\%)=$ Weight of microspheres $\times 100 /$ Total solids (polymers + PTH)

The hPTH encapsulation efficiency (EE) of microspheres was determined in supernatant after centrifugation at $50,000 \mathrm{rpm}$ for $2 \mathrm{~h}$ at $10^{\circ} \mathrm{C}$ using the hPTH ELISA kit (PH 1-34) according to the manufacturer's instructions, and calculated as follows:

$\mathrm{EE} \%=($ Total amount of PTH - FreePTH $) \times 100 /$ Total amount of PTH

The particle size and size distribution (polydispersity index, PDI) of microspheres were determined by photon correlation spectroscopy (Nano-ZS, Malvern Instruments, Malvern, UK) at $25^{\circ} \mathrm{C}(\mathrm{n}=3)$.

The zeta potential of microspheres was determined using electrophoretic light-scattering (Zetasizer Nano-ZS, Malvern Instruments, Malvern, UK) $(n=6)$.
The morphology of microspheres was investigated by scanning electron microscopy (SEM; FEI, QuantaTM FEG 450, Hillsboro, USA) at $25 \mathrm{kV}$.

\section{Gelation temperature of poloxamer solutions}

The sol-gel transition of poloxamer solutions at concentrations between $18 \%$ and $22 \%$ was measured using the Brookfield RVDV-II+ viscometer (Brookfield, Middleboro, USA) at temperatures between $14-37^{\circ} \mathrm{C}$.

\section{Preparation of thermosensitive poloxamer gel containing hPTH}

Poloxamer (20 mg; Pluronic ${ }^{\circledR}$ F127) was accurately weighed and dissolved in $100 \mathrm{~mL}$ distilled water at $5^{\circ} \mathrm{C}$ with stirring in a magnetic stirrer at $400 \mathrm{rpm}$ for $2 \mathrm{~h}$. Then, $80 \mu \mathrm{l}$ Forsteo was mixed with $320 \mu \mathrm{l}$ of the resulting poloxamer solution under the same stirring conditions for $5 \mathrm{~min}$. The final solution, containing $\mathrm{hPTH}$ at a concentration of $0.05 \mu \mathrm{g} / \mu \mathrm{L}$, was stored in a refrigerator at ${ }^{\circ} \mathrm{C}$ for $24 \mathrm{~h}$ to ensure complete dissolution, and then sterilized by filtration through a $0.22-\mu \mathrm{m}$ polyether sulphone membrane filter and stored at $4^{\circ} \mathrm{C}$ until use.

Preparation of thermosensitive poloxamer gel containing hpth-loaded chitosan microspheres

hPTH-loaded chitosan microspheres were dispersed in $20 \%(\mathrm{w} / \mathrm{v})$ poloxamer solution at $5^{\circ} \mathrm{C}$ with stirring at $200 \mathrm{rpm}$ for $2 \mathrm{~h}$ to achieve a final concentration of particles equivalent to $0.233 \mu \mathrm{g} / \mu \mathrm{L}$ hPTH.

\section{In vitro hPTH release}

In vitro hPTH release studies were performed using dialysis. A total of $10 \mathrm{mg}$ hPTH-loaded chitosan microspheres, suspended in $1 \mathrm{~mL}$ phosphate-buffered saline (PBS; $\mathrm{pH} 7.4$ ), and poloxamer gels containing hPTH-loaded chitosan microspheres equivalent to $10 \mathrm{mg}$ microspheres, were transferred separately into dialysis bags immersed in $9 \mathrm{~mL}$ PBS at $37^{\circ} \mathrm{C}$. At predetermined time intervals, $2 \mathrm{~mL}$ of the released medium was removed from outside the dialysis bags and replaced with $2 \mathrm{~mL}$ fresh buffer solution. The concentration of hPTH released was determined using the hPTH ELISA kit. 


\section{In vivo studies}

The present study was approved by the Committee of Animal Research and Ethics of Istanbul University, Turkey (2014/50). Fourty-eight adult female Sprague-Dawley rats, with an average age of 6 weeks and weighing 225-250 g, were used in this study. The rats were kept in an environment with a controlled temperature and 12-hour light/dark cycle, with food and water supplied ad libitum.

An animal model was chosen to test hPTH treatments applied after dental extractions in bisphosphonate-treated rats; to do this, we followed the MRONJ-like disease model given by Barba-Recreo et al., ${ }^{25}$ which replicates the histologic findings of the human disease. According to this MRONJ model, zoledronic acid (ZA) was chosen because of its high relative potency. The dosage and duration of ZA injections were administered as described by Basi et al. ${ }^{26} \mathrm{~A}$ dose of $2.25 \mathrm{mg} / \mathrm{kg}$ ZA (Zometa, $4 \mathrm{mg}$ flacon, Novartis, 87935, Turkey), which is comparable to the average accumulated dose in MRONJ patients, was administered daily by intraperitoneal injection to Sprague-Dawley rats over a 3-week period. The accumulated dose was obtained using a total of 21 doses. Thereafter, the rats were anesthetized by an intraperitoneal injection of $0.1 \%$ pentobarbital $(2.5 \mathrm{mg} / 100 \mathrm{~g}$ body weight), and the three left upper molars were extracted with dental elevators and forceps under local anesthesia with $1 \%$ lidocaine $(0.5 \mathrm{mg} / 100 \mathrm{~g}$ body weight) in the vestibular area. After tooth extraction, the rats were randomly assigned into four groups as follows:

a. In the no treatment group $(\mathrm{n}=12)$, rats were allowed to recover with no treatment.

b. In the negative control group ( $=12$ ), $30 \mu \mathrm{l}$ poloxamer hydrogel containing empty microspheres were immediately applied to the subperiosteum with a Hamilton microsyringe.

In treatment group- $1(\mathrm{n}=12)$, a local injection of poloxamer hydrogel containing hPTH at a concentration of $0.05 \mu \mathrm{g} / \mu \mathrm{L}$ was immediately applied to the subperiosteum with a Hamilton microsyringe. Then, intermittent injections of the poloxamer hydrogel containing the same concentration of hPTH were performed every 3 days.
In treatment group- $2(n=12)$, rats were treated with a single local injection of poloxamer hydrogel containing hPTH-loaded microspheres (equivalent to $0.233 \mu \mathrm{g} / \mathrm{L} \mathrm{hPTH}$ ) with a Hamilton microsyringe immediately after tooth extraction.

On the 10th and 21th days after tooth extraction, 6 rats from each experimental group were sacrificed by decapitation under general anesthesia. The hemimandibles were then harvested for further histomorphometrical analysis.

\section{Histomorphometrical analysis}

The rat heads were fixed in $10 \%$ buffered formalin solution. Samples were then decalcified with $25 \%$ formic acid. After decalcification, maxillas were desiccated from the extraction area in a sagittal plane. Cross-sections were processed for paraffin embedding, and histologic sections with a $4-\mu \mathrm{m}$ thickness were cut along the sagittal plane. Finally, the sections were stained with hematoxylin and eosin (H\&E). Histologic analysis was performed on all samples by a single blinded pathologist. After the sections were evaluated under a light microscope (Eclipse E600; Nikon, Tokyo, Japan), necrotic bone tissues with empty spaces of osteocytes and the formation of new bone with osteoblastic rimming were examined by histomorphometric assessment using the AnalySIS Five (Olympus Soft Imaging System, Münster, Germany) imaging analysis system. Images of all histological specimens were captured with a digital camera attached to a light microscope (Olympus Bx51; Olympus Corporation, Tokyo, Japan). Imaging software (Olympus DP71; Olympus Corporation, Tokyo, Japan) was used for histomorphometric analysis. The area representing both new bone formation and necrotic tissues was expressed in $\mathrm{mm}^{2}$ on photomicrographs (40x magnification).

\section{Statistical analysis}

Statistical analysis was conducted using the SPSS Statistics 22 software package (IBM Corp., NY, USA). Data obtained from each in vitro experiment, as well as the relationships between zeta potentials and particle sizes, were subjected to statistical analysis using one-way analysis of variance (ANOVA) followed by the Newman-Keuls multiple comparisons test 
to compare data between groups. $\mathrm{p}<0.05$ was considered statistically significant. In vivo data, whether the parameters showed normal distribution or not, were assessed using the Kolmogorov-Smirnov test. Intergroup comparisons of the parameters that showed normal distribution were performed using one-way ANOVA, and significant differences between groups were determined using Tukey's post hoc test. Intergroup comparisons of parameters that did not show normal distribution were performed using the Kruskal-Wallis test, and significant differences between groups were determined using the MannWhitney U test. Comparisons of the parameters between two groups showing normal distribution were performed using Student's $t$-test, and those between groups that did not show normal distribution were performed using the Mann-Whitney $U$ test. Significance was set at $\mathrm{p}<0.05$. All $\mathrm{p}$ values are two-sided.

\section{Results}

\section{In vitro studies results}

\section{Preparation and characterization of \\ PTH-loaded microspheres}

hPTH-loaded chitosan microspheres with different chitosan:TPP ratios (3:1 and 4:1) were prepared by ionotropic gelation, which is based on electrostatic interactions between a positively charged polymer (chitosan) and a negatively charged polyanion (TPP), using aqueous solutions of polymer and TPP at five different concentrations and two different chitosan:TPP mass ratios. The production yield, encapsulation efficiency, particle size, and size distribution of particles are shown in Table.
The production yield varied depending on the ratios between the cationic polymer and anionic TPP, as well as on the total mass of the solids. The production yields of microspheres ranged between $59.4 \%$ and $80.2 \%$, and increased with a decreasing chitosan:TPP mass ratio $(p<0.05)$. The highest production yield was obtained with the formulation prepared at a 3:1 polymer: TPP ratio with the lowest chitosan and TPP concentrations $(F K 5=80.2 \%)(p<0.05)$. The size of microsphere particles ranged from $0.61-4.90 \mu \mathrm{m}$. The smallest particles were obtained with FK5. An increase in the concentration of both the polymer and polyanion solutions significantly increased particle size and the size distribution (polydispersity) of the particles $(p<0.05)$. The particle size also increased with an increasing chitosan:TPP mass ratio $(\mathrm{p}<0.05)$. With an increased total mass of chitosan and TPP but at a fixed mass ratio of chitosan:TPP, an increase in size distribution was observed, as well as an increase in particle size from $0.81 \mu \mathrm{m}$ to $4.90 \mu \mathrm{m}$ for the formulations prepared at a ratio of $4: 1$, and an increase from $0.61 \mu \mathrm{m}$ to $1.04 \mu \mathrm{m}$ for those prepared at a 3:1 ratio. The polydispersity index (PDI) indicates the dispersion homogeneity at a range between 0 and 1. A PDI approaching 0 indicates a more homogenous dispersion. ${ }^{27}$ A decrease in the concentration of chitosan and/or TPP dramatically reduced PDI in this study. For instance, although the FK2 and FK3 formulations had the same mass ratio (4:1), FK3 showed a much lower PDI than FK2 ( $<<0.05)$, as shown in Table 1. These finding are correlated with those of Hu et al. ${ }^{28}$

The zeta potential is critical in understanding the surface charge characteristics of microspheres. A higher zeta potential results in greater electrostatic repulsion forces between the particles, which leads to greater

Table. The production yield, encapsulation efficiency, particle size, and size distribution of hPTH-loaded chitosan microspheres ( $\mathrm{n}=3$ ).

\begin{tabular}{lcccccc} 
Formulation & $\begin{array}{c}\text { Chitosan:TPP mass ratio } \\
\text { (percentage) }\end{array}$ & $\begin{array}{c}\text { Production yield } \\
(\%)\end{array}$ & $\begin{array}{c}\text { Particle size } \\
(\mu \mathrm{m} \pm \text { SD) }\end{array}$ & $\begin{array}{c}\text { Polydispersity Index } \\
(\text { PDI) }\end{array}$ & $\begin{array}{c}\text { Zeta potential } \\
(\mathrm{mV} \pm \text { SD) }\end{array}$ & $\begin{array}{c}\text { Encapsulation } \\
\text { efficiency }(\%)\end{array}$ \\
\hline FK1 & $4: 1(0.5: 0.125)$ & $59.4 \pm 5.7$ & $4.90 \pm 0.82$ & 0.750 & $+18.2 \pm 0.4$ & $44.2 \pm 2.13$ \\
FK2 & $4: 1(0.25: 0.25)$ & $62.1 \pm 6.3$ & $1.60 \pm 0.21$ & 0.685 & $+16.7 \pm 0.6$ & $39.6 \pm 3.08$ \\
FK3 & $4: 1(0.25: 0.125)$ & $64.2 \pm 5.6$ & $0.81 \pm 0.19$ & 0.354 & $+16.5 \pm 0.8$ & $34.1 \pm 2.67$ \\
FK4 & $3: 1(0.25: 0.17)$ & $76.3 \pm 5.9$ & $1.04 \pm 0.09$ & 0.618 & $+17.8 \pm 1.1$ & $74.8 \pm 5.65$ \\
FK5 & $3: 1(0.25: 0.125)$ & $80.2 \pm 6.3$ & $0.61 \pm 0.08$ & 0.286 & $+18.5 \pm 0.6$ & $72.9 \pm 4.84$ \\
\hline
\end{tabular}

$p<0.05$ was considered as significant. 
separation of particles, reducing the extent of aggregation caused by Van de Waals interactions. ${ }^{29}$ In this study, microsphere formulations resulted in positive zeta potential value of between $+16.5 \mathrm{mV}$ and $+18.7 \mathrm{mV}$; the polymer:TPP mass ratio did not significantly influence the surface charge of the microspheres.

Particle morphology was evaluated by SEM. As seen in Figure 1, hPTH-loaded microspheres presented a uniform spherical morphology.

The effect of the chitosan:TPP mass ratio on encapsulation efficiency was investigated at ratios of 3:1 and 4:1. As presented in Table 1, the encapsulation efficiency decreased from $72.9 \%$ to $34.1 \%$ as the mass ratio increased from 3:1 (FK5) to 4:1 (FK3) (p<0.05). An increase in the concentration of both polymer and polyanion solutions at the same mass ratios slightly increased the encapsulation efficiency of the microspheres $(p>0.05)$. The reason for the slight increase may have been the use of low-concentration chitosan (0.25-0.5\%) and low-concentration TPP (0.125-0.25\%). Additionally, previous findings have shown that high concentrations of chitosan $(>3 \mathrm{mg} / \mathrm{ml})$ dramatically decreased encapsulation efficiency and induced aggregation. ${ }^{30}$ Following our optimization and characterization studies on the hPTH-loaded microspheres, the most efficient hPTH carrier system was as follows: microspheres at a chitosan:TPP mass ratio of 3:1 and the lowest polymer and polyanion concentration (FK5); this resulted in an optimal particle diameter of $0.61 \mu \mathrm{m}$, narrow particle size distribution $(0.286)$, high zeta potential value $(+18.5 \mathrm{mV})$, and high encapsulation efficiency $(72.9 \%)$.

\section{Optimisation of poloxamer gel formulations}

The sol-gel transition point, at which the rheological properties of a substance change from a liquid-like state to solid-like state, of implantable thermoreversible systems-such as poloxamer formulations-should be around body temperature to ensure that the formulation can be easily applied to the local area in a liquid-like state, and to improve its retention time in a solid-like state within the application site. ${ }^{31}$ Poloxamer F127 has a liquid-like state at low temperatures due to excessive hydrogen bonds between the water molecules and oxygen with the polymer, and is converted into a high-viscosity gel at body temperature. ${ }^{32}$ In this study, different concentrations of poloxamer in water (between $18 \%$ and $22 \%$ ) were prepared at $10^{\circ} \mathrm{C}$, and their sol-gel transitions were measured using a viscometer at temperatures of $14-37^{\circ} \mathrm{C}$ to determine the ideal concentration for use at lower temperatures with long-term retention in the subperiosteal cavity at approximately body temperature. Figure 2 demonstrates the results of

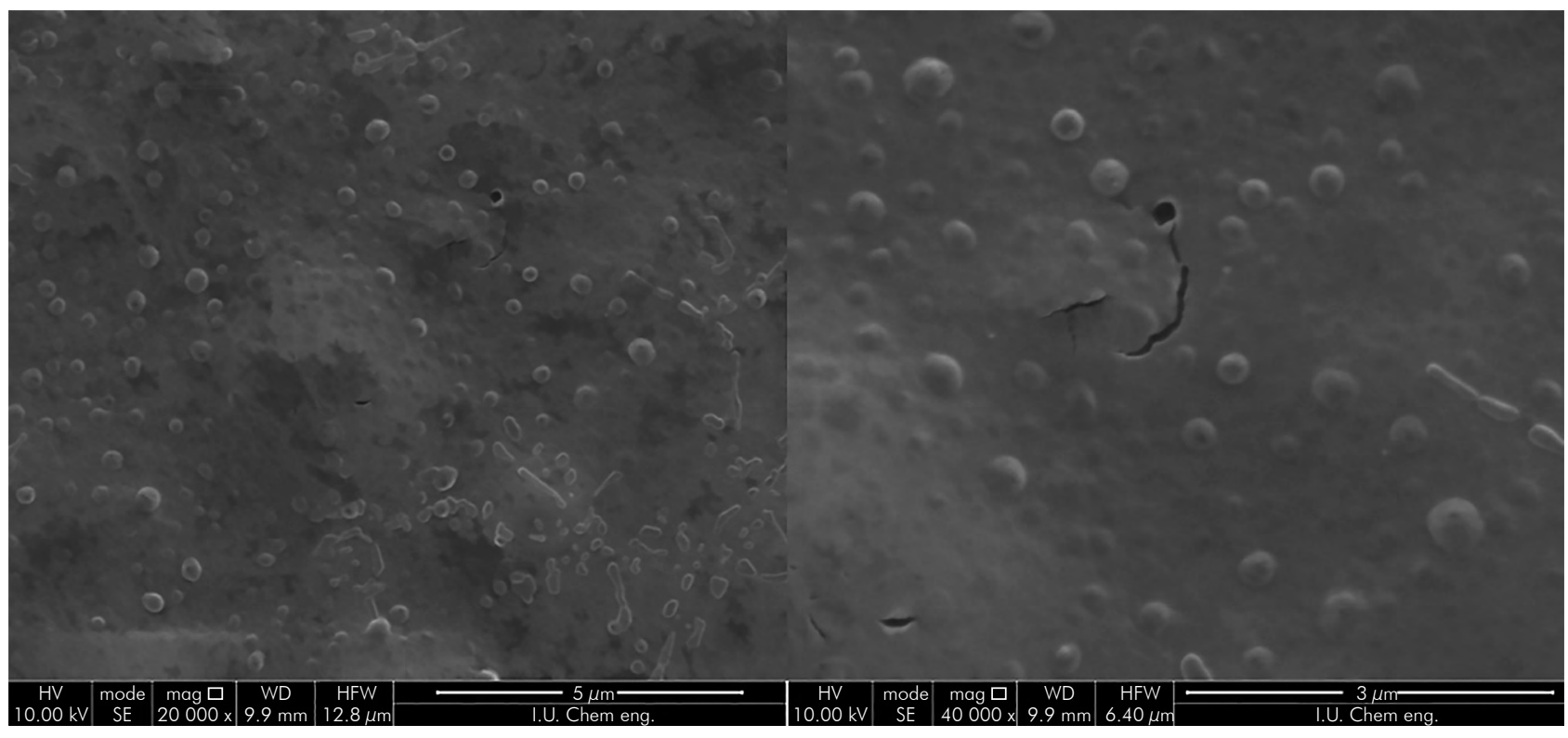

Figure 1. Morphological characteristics of hPTH-loaded chitosan microspheres using scanning electron microscopy. 
the viscosity studies of the poloxamer hydrogels prepared with polymer concentrations of between $18 \%$ and $22 \%$.

The viscosity of the poloxamer hydrogels was affected by the concentration of the polymer and by the ambient temperature. While the viscosity at all the concentrations (between $18 \%$ and $22 \%$ ) did not significantly change at temperatures up to $20^{\circ} \mathrm{C}$ $(100-179 \mathrm{cP})$, the viscosity began to increase rapidly between $20-27^{\circ} \mathrm{C}$; the rate of increase in viscosity then slowed down between $27-36^{\circ} \mathrm{C}$ at all poloxamer concentrations (Figure 3). At body temperature $\left(37^{\circ} \mathrm{C}\right)$, there was no significant difference in the viscosity of poloxamer hydrogels prepared at concentrations between $20 \%(3,121 \mathrm{cP})$ and $22 \%(3,234 \mathrm{cP})(\mathrm{p}>0.05)$. A poloxamer hydrogel concentration of $20 \%$ was thus chosen to be the most efficient for the implantable sustained-release carrier system for $\mathrm{hPTH}$ and $\mathrm{hPTH}-$ loaded microspheres.

\section{In Vitro hPTH release}

The drug-release behaviors of microsphere and hydrogel formulations were measured in vitro using a dialysis membrane at $\mathrm{pH} 7.4$ and $37^{\circ} \mathrm{C}$ (Figure 3). Poloxamer hydrogel, containing a free $\mathrm{hPTH}$ formulation, showed the fastest release rate; $\mathrm{hPTH}$ was completely released from this formulation within 5 days. However, a marked reduction in drug release was observed when hPTH was incorporated into microspheres. Around $57 \%$ and $43 \%$ of hPTH from

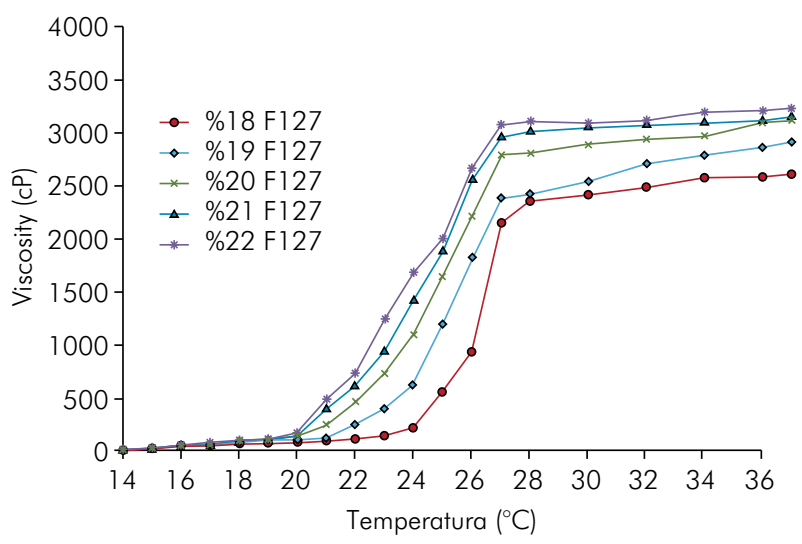

Figure 2. The viscosity of poloxamer hydrogels prepared with different polymer concentrations of between $18 \%$ and $22 \%$ at different temperatures. $p<0.05$ was considered as significant. microspheres and poloxamer hydrogels containing microspheres, respectively, were released in the first week, then the release rate slowed and was completed after 21 and 27 days from the hPTH-loaded microspheres and the poloxamer hydrogel containing hPTH-loaded microspheres, respectively. As seen in Figure 3, microspheres and poloxamer/microsphere combinations significantly extended the release of $\mathrm{hPTH}$ from formulations.

\section{In Vivo studies results}

\section{Histomorphometric results}

As shown in Figure 4, no statistically significant differences were found in the day 10 necrosis levels between the groups ( $\mathrm{p}: 0.392 ; \mathrm{p}>0.05$ ), but a statistically significant difference was detected in the day 21 necrosis levels between different groups (p: $0.0001 ; p<0.01$ ). The level of necrosis on day 21 was found to be significantly higher in the no treatment group ( $<0.01$ ) compared to group 1 (p: 0.004) and group 2 (p: 0.004). The level of necrosis on day 21 was significantly higher in the negative control group compared to group 2 (p: 0.016; $p<0.05$ ), but there was no significant difference between group 1 and group 2.

Intragroup analysis indicated that the decrease in necrosis level observed on day 21 was significantly lower than the level of necrosis on day 10 in group 1 and group 2 (p: 0.004; $p<0.01$ ). In negative control

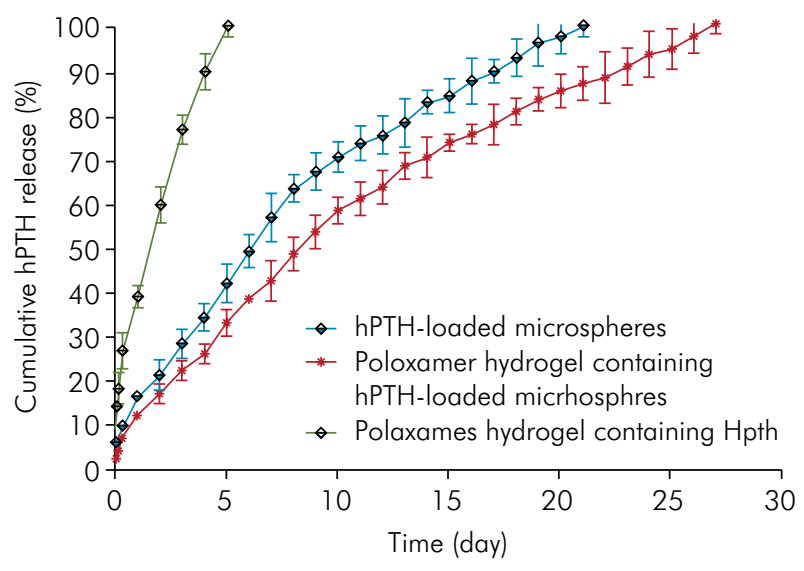

Figure 3. In vitro hPTH release profiles from the microspheres and poloxamer hydrogel formulations $(n=3)$. 
group (p: $0.078 ; \mathrm{p}>0.05$ ) and no treatment group (p: $0.262 ; p>0.05$.$) , no statistical change was observed$ in the levels of necrosis on day 21 compared to that observed on day 10.

As shown in Figure 5, there was a significant difference in the levels of new bone formation at day 10 across all groups (p: 0.003; $p>0.01$ ). The level of new bone formation on day 10 was found to be significantly higher in group $2(\mathrm{p}<0.01)$ compared to the negative control (p: 0.007) and no traetment groups (p: 0.007). Furthermore, there was no statistically significant difference between the negative control and no treatment groups with regards to the level of new bone formation on day 10 ( $p>0.05)$. Besides, there was a statistically significant difference between the levels of new bone formation on day 21 across all groups (p: $0.001 ; p<0.01)$. The level of new bone formation on day 21 was found to be significantly lower in the no treatment group $(\mathrm{p}<0.01)$ compared to group 1 (p: 0.002), group 2 (p: 0,002), and the negative control group (p: 0.007). Finally, there was no statistically significant difference between group 1 and group 2 in terms of the level of new bone formation on day 21 ( $p>0.05)$.

Intragroup analysis indicated that the observed increase in the new bone formation levels on day 21 was statistically significant compared to that observed

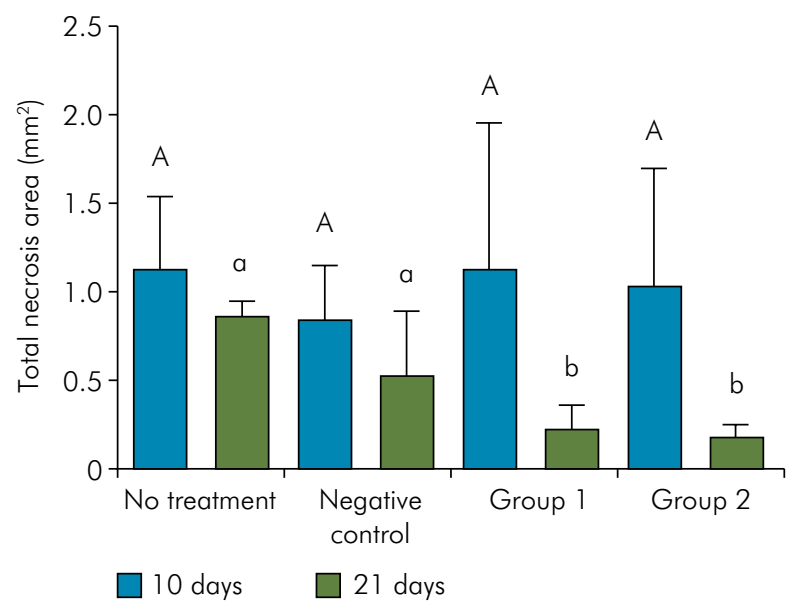

Figure 4. Extent of bone necrosis in different groups after the indicated time periods $(p<0.05$ was considered as significant). Upper-case letters indicate significant differences between necrosis on 10 days. Lower-case letters indicate significant differences between necrosis of 21 days. on day 10 in group 1 (p: 0.016; $\mathrm{p}<0.05)$; this was not observed in group 2 (Figure 6).

\section{Discussion}

Bone healing comprises a sequence of complex biological processes. Bisphosphonates are known to severely inhibit the biological processes of bone healing after invasive dental procedures such as tooth extraction, periodontal surgery, and related interventions. ${ }^{33}$ There are several suggested models regarding the pharmacokinetic profile of bisphosphonates. ${ }^{34}$ On the other hand, the effective treatment due to bisphosphonate uptake has many challenges. ${ }^{35}$ MRONJ was first described by Marx and Stern, ${ }^{36}$ named BRONJ (bisphosphonate related osteonecrosis of the jaw) back then, as an outcome of an unhealed exposed bone, where symptoms worsened and bone necrosis quantitatively increased with debridement. Today, in addition to the scientific discussions regarding its pathogenesis, jawbone osteonecrosis also remains a clinical problem whose successful treatment is a challenge for dentistry clinics.

$\mathrm{ZA}$, a nitrogen-containing bisphosphonate most commonly associated with osteonecrosis, is a widely preferred drug for animal models; it has previously been used to investigate the pathophysiology of

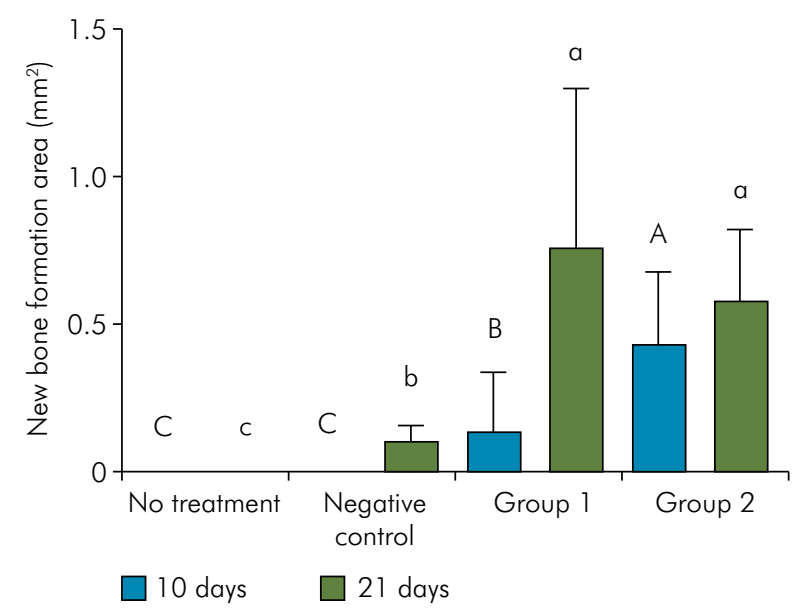

Figure 5. The distribution of new bone formation in different groups after the indicated time periods. ( $p<0.05$ was considered as significant). Upper-case letters indicate significant differences between new bone formation on 10 days. Lower-case letters indicate significant differences new bone formation on 21 days. 

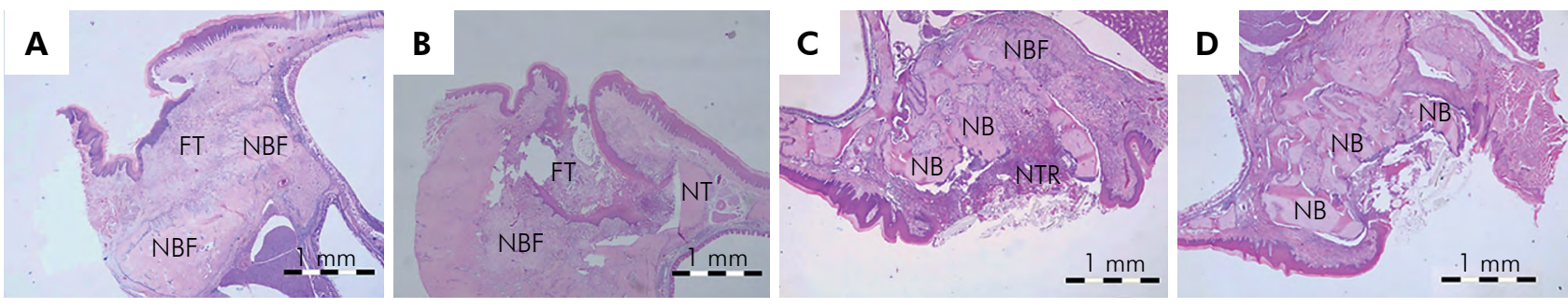

Figure 6. Histomorphometric analysis on day 21 after treatment. A) New bone formation between fibrous tissue in the extraction area in experimental group 1 (H\&E x 40). B) New bone formation under the fibrous tissue in group 2 (H\&E x 40). C) Negative control group. Extraction socket filled with necrotic tissue remains, as well as necrotic bone; new bone formation was observed at the extraction socket (H\&E x 40). D) No treatment control group. Necrotic tissue remained within a large area surrounding the necrotic bone (H\&E x 40). FT, Fibrous tissue; NBF, New bone formation; NB, New bone; NT, Necrotic Tissue, NTR, necrotic tissue remnants.

MRONJ, to determine strategies to prevent it, and to develop therapeutic methods to address this problem. ${ }^{25}$

In previous publications using animal models, ${ }^{37-39}$ substantial inconsistencies were observed depending on the species of laboratory animal used, the administration method, the dosage and duration of bisphosphonate treatment, and the existence of predisposing factors. Currently, there is no standard animal model to investigate the formation of MRONJ.

Ideally, the animal model for MRONJ should simulate the clinical presentation of osteonecrosis in the lesions that occur following a bisphosphonate treatment protocol administered in a manner identical to that in humans. Specifically, an animal model should present with jawbone exposure that has not healed for 8 weeks in a subject treated with bisphosphonate without radiation treatment. Although exposed bone was observed in all the rats used in our study, a histopathological evaluation was conducted to compare the size of the necrotic areas across the experimental groups and to investigate the function of the parathyroid hormone formulation used. Biasotto et al. ${ }^{38}$ reported that no standardization was found in terms of the dose of ZA used in animal models and re-established the optimal concentration of ZA by considering the dose used to treat hematological diseases in humans, as well as the differences in bone metabolism observed between the two species.

In our study, the administered dose was determined based on the information that in humans, MRONJ occurred after the administration of ZA at a dose of $4 \mathrm{mg} / \mathrm{month}$. Accordingly, the equivalent dose for a rat was calculated as $2.25 \mathrm{mg} / \mathrm{kg} .{ }^{26,40} \mathrm{The} \mathrm{ZA}$ administration protocol, which has previously been employed in other studies, is based on the estimation that a daily dose of $0.11 \mathrm{mg} / \mathrm{kg}$ for 21 days is required to reach the dose of $2.25 \mathrm{mg} / \mathrm{kg}$ that is necessary for the onset of MRONJ. ${ }^{6,15,28-29,53}$ Therefore, the animal model used in our study was treated with $0.11 \mathrm{mg} / \mathrm{kg}$ ZA daily for 21 days intraperitoneally.

Several studies have examined the effect of PTH on craniofacial bone healing in animal models. ${ }^{16,41,42,43}$ Andreassen and Cacciafesta ${ }^{16}$ intermittently administered PTH via the systemic route in combination with guided bone regeneration in an animal model. They reported that the group treated with PTH $(60 \mu \mathrm{g} / \mathrm{kg})$ for 35 days showed significantly higher rates of new bone formation compared to the untreated group. There are also studies in which PTH was locally administered to increase bone regeneration. ${ }^{41,42,43}$ Jung et al. ${ }^{41}$ used a PTH-loaded polyethylene glycol matrix vector combined with bone grafts containing hydroxyapatite-tricalcium phosphate, and their study concluded that locally administered PTH $(20 \mu \mathrm{g} / \mathrm{mL})$ in combination with bone grafts led to a significantly greater stimulation of bone regeneration. In another study by Jung et al., ${ }^{42}$ a PTH-loaded polyethylene glycol matrix $(20 \mu \mathrm{g} / \mathrm{mL})$ was inserted in the space formed around an implant with autogenous bone. Although higher rates of new bone formation were found in the group with autogenous bone administration only, they reported that new bone formation was also high in the group with a PTH-loaded polyethylene glycol matrix, which was comparable to the autogenous bone only group. Similarly, Valderrama et $a{ }^{43}$ assessed new 
bone formation at 2 and 4 weeks after implantation of a PTH-loaded glycol matrix; the PTH-loaded polyethylene glycol group also yielded better results in their study. In our study, the presence of newly formed bone around the defect area was also significantly higher in the group- 1 and 2 than in the other groups in accordance with all the literature mentioned above.

Because our study aimed to demonstrate the anabolic effect of PTH on bone formation, we performed repeated injections of $1 \mu \mathrm{g} \mathrm{PTH}$ subperiosteally through the palatal region using micro injectors every 3 days for 21 days in the treatment group-1. In the treatment group- 2, $7 \mu \mathrm{g}$ $\mathrm{PTH}$, the dose to which group- 1 was exposed over the course of 21 days, was administered at once. In both groups, the increase in new bone formation observed on days 10 and 21 was statistically significant compared to the other groups.

To attenuate the symptoms of MRONJ, intermittent administration of low dose PTH was previously proposed by Harper and Fung. ${ }^{17}$ They concluded that injecting PTH at a dose of $0.3 \mathrm{mg} / \mathrm{kg} /$ day for 6 months yielded successful results in the treatment of MRONJ. Subsequently, many other case reports have been published investigating the role of intermittent systemic PTH administration to treat MRONJ. In all previous studies in which low dose PTH was intermittently administered to treat MRONJ, the attenuation of MRONJ symptoms was achieved by administering PTH via the systemic route. ${ }^{44,45}$ To our knowledge, there are no studies in which PTH was locally administered with a sustained releasing vector for the prevention of occurrence or reducing the extent of MRONJ. In this study, the decrease in necrotic bone area in locally administered PTH groups was consistent with previous reports where PTH was systemically administered.

Local therapy has many advantages, such as avoiding potential adverse effects of systemic therapy, a decreased dosage, and effective maintenance of adequate concentrations of the drug locally. ${ }^{13}$ For this reason, in our study, we designed a drug formulation for local PTH treatment to lead to a localized anabolic effect. We conducted the in-vitro study according with several previously designed drug delivery systems to achieve comparable results, such as those of Wei et al. ${ }^{46}$ who formulated poly (lactic-co-glycolic acid) microspheres controlling the release of PTH, and Liu et al., ${ }^{47}$ who developed an implantable carrying system that contains alternating polyanhydride isolation layers and PTH-loaded alginate layers. Narayanan et al. ${ }^{48}$ also developed an intravenously administered PTH-loaded chitosan nanoparticle system.

In our study, in group- 1, where PTH was administered with repeated injections, poloxamer (Pluronic F127) was chosen as a polymeric vector to ensure the localization of hPTH to the target area. The inert nature of the poloxamer was demonstrated previously by Issa et al., ${ }^{49}$ therefore, in the present study we decided that sacrificing an extra 12 more rats was unnecessary, and the study did not consist a control group treated with the poloxamer alone. In group- 2, where PTH was administered through a single injection, chitosan was preferred as a polymer for the preparation of the hPTH-loaded microspheres. Chitosan is a biocompatible, biodegradable, and non-toxic polysaccharide with high loading capacity, which is approved by the FDA for use in the biomedical fields. Chitosan has been commonly used as the main material for the production of therapeutic protein molecules, as well as for a nonviral gene delivery vector. ${ }^{48}$

We also aimed to increase the efficacy of hPTH by reducing the size of the chitosan particles to ensure the sustained release of hPTH. Thus, a single injection of PTH-loaded chitosan microspheres was administered to compare the efficacy of this treatment against that given to group 1. Comparing this drug formulation prepared in our study to the standard regimen of repeated injections on day 21 in terms of new bone formation, no significant differences were observed between group 1 and group 2, indicating that the drug applied through a single injection in group 2 was still efficient on day 21, despite a decrease in the release rate after 10 days in group 1. This result suggests that the two groups showed similar efficiency in terms of new bone formation.

This study tried to analyze local efficacy of $\mathrm{hPTH}$ thoroughly to indicate the reduction of the necrotic bone area. The limitations of the study can be listed as small sample size and lack of analysis 
with immunohistochemistry technique. Future research is needed with higher sample size with immunohistochemical observation. Even with the limitations of the present study, to the best of our knowledge, this is the first study to investigate the efficacy of local application method with the sustained-release microspheres to attenuate of MRONJ.

\section{Conclusion}

In our study, it was observed that in rats that were given therapeutic doses of systemic bisphosphonates, locally administered hPTH stimulated bone healing. Based on our results, we can conclude that the drug formulations administered as single or repeated injections showed similar effects in terms of bone healing and the attenuation of necrosis. Thus, for the ideal clinical adaptation of these drugs, we believe that a single injection of hPTH in a sustained release formulation would be preferable to ensure patient compliance and comfort.

\section{Acknowledgments}

This work was supported by Scientific Research Projects Coordination Unit of Istanbul University [Project Number: 44564]. The present work was performed with the approval of the Animal Rights Committee of Istanbul University (Grant number: 2014/50).

\section{References}

1. Papapoulos SE. Bisphosphonates: how do they work? Best Pract Res Clin Endocrinol Metab. 2008 Oct;22(5):831-47. https://doi.org/10.1016/i.beem.2008.07.001

2. Sharma D, Hamlet S, Petcu E, Ivanovski S. Animal models for bisphosphonate-related osteonecrosis of the jaws: an appraisal. Oral Dis. 2013 Nov; 19(8):747-54. https://doi.org/10.1111/odi.12067

3. Roelofs AJ, Thompson K, Gordon S, Rogers MJ. Molecular mechanisms of action of bisphosphonates: current status. Clin Cancer Res. 2006 Oct;12(20 Pt 2):6222s-30s. https://doi.org/10.1158/1078-0432.CCR-06-0843

4. Dimitrakopoulos I, Magopoulos C, Karakasis D. Bisphosphonate-induced avascular osteonecrosis of the jaws: a clinical report of 11 cases. Int J Oral Maxillofac Surg. 2006 Jul;35(7):588-93. https://doi.org/10.1016/i.ijom.2006.02.022

5. Senel FC, Kadioglu Duman M, Muci E, Cankaya M, Pampu AA, Ersoz S, et al. Jaw bone changes in rats after treatment with zoledronate and pamidronate. Oral Surg Oral Med Oral Pathol Oral Radiol Endod. 2010 Mar;109(3):385-91. https://doi.org/10.1016/i.tripleo.2009.10.011

6. Huja SS, Fernandez SA, Phillips C, Li Y. Zoledronic acid decreases bone formation without causing osteocyte death in mice. Arch Oral Biol. 2009 Sep;54(9):851-6. https://doi.org/10.1016/j.archoralbio.2009.06.002

7. Ruggiero SL, Dodson TB, Fantasia J, Goodday R, Aghaloo T, Mehrotra B, et al. Medication-related osteonecrosis of the jaw-2014 update. J Oral Maxillofac Surg. 2014;72(10):1938-56. https://doi.org/10.1016/i.joms.2014.04.031

8. Aghaloo TL, Kang B, Sung EC, Shoff M, Ronconi M, Gotcher JE, et al. Periodontal disease and bisphosphonates induce osteonecrosis of the jaws in the rat. J Bone Miner Res. 2011 Aug;26(8):1871-82. https://doi.org/10.1002/jbmr.379

9. Maahs MP, Azambuja AA, Campos MM, Salum FG, Cherubini K. Association between bisphosphonates and jaw osteonecrosis: a study in Wistar rats. Head Neck. 2011 Feb;33(2):199-207. https://doi.org/10.1002/hed.21422

10. Kim JW, Kim SJ, Kim MR. Leucocyte-rich and platelet-rich fibrin for the treatment of bisphosphonate-related osteonecrosis of the jaw: a prospective feasibility study. Br J Oral Maxillofac Surg. 2014 Nov;52(9):854-9. https://doi.org/10.1016/i.bjoms.2014.07.256

11. Vescovi P. Bisphosphonates and osteonecrosis: an open matter. Clin Cases Miner Bone Metab. 2012 Sep;9(3):142-4.

12. Cicciù M, Herford AS, Juodžbalys G, Stoffella E. Recombinant human bone morphogenetic protein type 2 application for a possible treatment of bisphosphonates-related osteonecrosis of the jaw. J Craniofac Surg. 2012 May;23(3):784-8. https://doi.org/10.1097/SCS.0b013e31824dbdd4

13. Chan HL, McCauley LK. Parathyroid hormone applications in the craniofacial skeleton. J Dent Res. 2013 Jan;92(1):18-25. https://doi.org/10.1177/0022034512464779

14. Jilka RL. Molecular and cellular mechanisms of the anabolic effect of intermittent PTH. Bone. 2007 Jun;40(6):1434-46. https://doi.org/10.1016/i.bone.2007.03.017

15. Dayisoylu EH, Şenel FÇ, Üngör C, Tosun E, Çankaya M, Ersöz S, et al. The effects of adjunctive parathyroid hormone injection on bisphosphonate-related osteonecrosis of the jaws: an animal study. Int J Oral Maxillofac Surg. 2013 Nov;42(11):1475-80. https://doi.org/10.1016/i.ijom.2013.05.001

16. Andreassen TT, Cacciafesta V. Intermittent parathyroid hormone treatment enhances guided bone regeneration in rat calvarial bone defects. J Craniofac Surg. 2004 May;15(3):424-7. https://doi.org/10.1097/00001665-200405000-00014 
- The efficacy of sustained-release chitosan microspheres containing recombinant human parathyroid hormone on MRONJ

17. Harper RP, Fung E. Resolution of bisphosphonate-associated osteonecrosis of the mandible: possible application for intermittent low-dose parathyroid hormone [rhPTH(1-34)] [rhPTH(1-34)]. J Oral Maxillofac Surg. 2007 Mar;65(3):573-80. https://doi.org/10.1016/i.joms.2006.10.076

18. Roberts GA. Solubility and solution behaviour of chitin and chitosan. In: Roberts GA, editor. Chitin Chemistry. UK: Macmillan Education; 1992. pp. 274-329. https://doi.org/10.1007/978-1-349-11545-7_6

19. Singla AK, Chawla M. Chitosan: some pharmaceutical and biological aspects—an update. J Pharm Pharmacol. 2001 Aug;53(8):1047-67. https://doi.org/10.1211/0022357011776441

20. Thanou M, Verhoef JC, Junginger HE. Oral drug absorption enhancement by chitosan and its derivatives. Adv Drug Deliv Rev. 2001 Nov;52(2):117-26. https://doi.org/10.1016/S0169-409X(01)00231-9

21. de Vasconcelos CL, Bezerril PM, dos Santos DE, Dantas TN, Pereira MR, Fonseca JL. Effect of molecular weight and ionic strength on the formation of polyelectrolyte complexes based on poly(methacrylic acid) and chitosan. Biomacromolecules. 2006 Apr;7(4):1245-52. https://doi.org/10.1021/bm050963w

22. Jeong B, Kim SW, Bae YH. Thermosensitive sol-gel reversible hydrogels. Adv Drug Deliv Rev. 2002 Jan;54(1):37-51. https://doi.org/10.1016/S0169-409X(01)00242-3 PMID:11755705

23. Desai SD, Blanchard J. In vitro evaluation of pluronic F127-based controlled-release ocular delivery systems for pilocarpine. J Pharm Sci. 1998 Feb;87(2):226-30. https://doi.org/10.1021/js970090e

24. López-León T, Carvalho EL, Seijo B, Ortega-Vinuesa JL, Bastos-González D. Physicochemical characterization of chitosan nanoparticles: electrokinetic and stability behavior. J Colloid Interface Sci. 2005 Mar;283(2):344-51. https://doi.org/10.1016/i.jcis.2004.08.186

25. Barba-Recreo P, Del Castillo Pardo de Vera JL, García-Arranz M, Yébenes L, Burgueño M. Zoledronic acid - related osteonecrosis of the jaws. Experimental model with dental extractions in rats. J Craniomaxillofac Surg. 2014 Sep;42(6):744-50. https://doi.org/10.1016/j.jcms.2013.11.005

26. Basi DL, Hughes PJ, Thumbigere-Math V, Sabino M, Mariash A, Lunos SA, et al. Matrix metalloproteinase-9 expression in alveolar extraction sockets of Zoledronic acid-treated rats. J Oral Maxillofac Surg. 2011 Nov;69(11):2698-707. https://doi.org/10.1016/i.joms.2011.02.065

27. Du J, Zhang S, Sun R, Zhang LF, Xiong CD, Peng YX. Novel polyelectrolyte carboxymethyl konjac glucomannan-chitosan nanoparticles for drug delivery. II. Release of albumin in vitro. J Biomed Mater Res B Appl Biomater. 2005 Feb;72(2):299-304. https://doi.org/10.1002/jbm.b.30156

28. Hu B, Pan C, Sun Y, Hou Z, Ye H, Zeng X. Optimization of fabrication parameters to produce chitosan-tripolyphosphate nanoparticles for delivery of tea catechins. J Agric Food Chem. 2008 Aug;56(16):7451-8. https://doi.org/10.1021/jf801111c PMID:18627163

29. Cevher E, Salomon SK, Makrakis A, Li XW, Brocchini S, Alpar HO. Development of chitosan-pullulan composite nanoparticles for nasal delivery of vaccines: optimisation and cellular studies. J Microencapsul. 2015;32(8):755-68. https://doi.org/10.3109/02652048.2015.1073392

30. Xu Y, Du Y. Effect of molecular structure of chitosan on protein delivery properties of chitosan nanoparticles. Int J Pharm. 2003 Jan;250(1):215-26. https://doi.org/10.1016/S0378-5173(02)00548-3

31. Aguilar MR, Elvira C, Gallardo A, Vázquez B, Román JS. Smart polymers and their applications as biomaterials. In: Ashammakhi N, Reis RL, Chiellini E, editors. Topics in tissue eng. 87935: Expertissues E-book; 2007. Vol.3, Chapter 6.

32. Galgatt UC, Chaudhari PD. Preformulation study of poloxamer 407 gels: effect of additives. Int J Pharma Sci. 2014;6(1):130-3.

33. Bagan L, Jiménez Y, Leopoldo M, Murillo-Cortes J, Bagan J. Exposed necrotic bone in 183 patients with bisphosphonate-related osteonecrosis of the jaw: associated clinical characteristics. Med Oral Patol Oral Cir Bucal. 2017 Sep;22(5):e582-5. https://doi.org/10.4317/medoral.22133

34. Kimmel DB. Mechanism of action, pharmacokinetic and pharmacodynamic profile, and clinical applications of nitrogen-containing bisphosphonates. J Dent Res. 2007 Nov;86(11):1022-33. https://doi.org/10.1177/154405910708601102

35. Kumar V, Sinha RK. Bisphosphonate related osteonecrosis of the jaw: an update. J Maxillofac Oral Surg. 2014 Dec;13(4):386-93. https://doi.org/10.1007/s12663-013-0564-x

36. Marx RE, Stern D. Oral and Maxillofacial Pathology: a rationale for treatment. Hanover Park: Quintessence; 2002.

37. Hikita H, Miyazawa K, Tabuchi M, Kimura M, Goto S. Bisphosphonate administration prior to tooth extraction delays initial healing of the extraction socket in rats. J Bone Miner Metab. 2009;27(6):663-72. https://doi.org/10.1007/s00774-009-0090-6

38. Biasotto M, Chiandussi S, Zacchigna S, Moimas S, Dore F, Pozzato G, et al. A novel animal model to study non-spontaneous bisphosphonates osteonecrosis of jaw. J Oral Pathol Med. 2010 May;39(5):390-6. https://doi.org/10.1111/i.1600-0714.2009.00878.x

39. Conte Neto N, Spolidorio LC, Andrade CR, S Bastos A, Guimarães M, Marcantonio E Jr. Experimental development of bisphosphonate-related osteonecrosis of the jaws in rodents. Int J Exp Pathol. 2013 Feb;94(1):65-73. https://doi.org/10.1111/iep.12007

40. Sonis ST, Watkins BA, Lyng GD, Lerman MA, Anderson KC. Bony changes in the jaws of rats treated with zoledronic acid and dexamethasone before dental extractions mimic bisphosphonate-related osteonecrosis in cancer patients. Oral Oncol. 2009 Feb;45(2):164-72. https://doi.org/10.1016/i.oraloncology.2008.04.013

41. Jung RE, Hämmerle $\mathrm{CH}$, Kokovic V, Weber FE. Bone regeneration using a synthetic matrix containing a parathyroid hormone peptide combined with a grafting material. Int J Oral Maxillofac Implants. 2007 Mar-Apr;22(2):258-66.

42. Jung RE, Cochran DL, Domken O, Seibl R, Jones AA, Buser D, et al. The effect of matrix bound parathyroid hormone on bone regeneration. Clin Oral Implants Res. 2007 Jun;18(3):319-25. https://doi.org/10.1111/j.1600-0501.2007.01342.x 
43. Valderrama P, Jung RE, Thoma DS, Jones AA, Cochran DL. Evaluation of parathyroid hormone bound to a synthetic matrix for guided bone regeneration around dental implants: a histomorphometric study in dogs. J Periodontol. 2010 May;81(5):737-47. https://doi.org/10.1902/jop.2010.090562

44. Kwon YD, Lee DW, Choi BJ, Lee JW, Kim DY. Short-term teriparatide therapy as an adjunctive modality for bisphosphonate-related osteonecrosis of the jaws. Osteoporos Int. 2012 Nov;23(11):2721-5. https://doi.org/10.1007/s00198-011-1882-9

45. Narváez J, Narváez JA, Gómez-Vaquero C, Nolla JM. Lack of response to teriparatide therapy for bisphosphonate-associated osteonecrosis of the jaw. Osteoporos Int. 2013 Feb;24(2):731-3. https://doi.org/10.1007/s00198-012-1918-9

46. Wei G, Pettway GJ, McCauley LK, Ma PX. The release profiles and bioactivity of parathyroid hormone from poly(lactic-co-glycolic acid) microspheres. Biomaterials. 2004 Jan;25(2):345-52. https://doi.org/10.1016/S0142-9612(03)00528-3

47. Liu X, Pettway GJ, McCauley LK, Ma PX. Pulsatile release of parathyroid hormone from an implantable delivery system. Biomaterials. 2007 Oct;28(28):4124-31. https://doi.org/10.1016/i.biomaterials.2007.05.034

48. Narayanan D, Anitha A, Jayakumar R, Nair SV, Chennazhi KP. Synthesis, characterization and preliminary in vitro evaluation of PTH 1-34 loaded chitosan nanoparticles for osteoporosis. J Biomed Nanotechnol. 2012 Feb;8(1):98-106. https://doi.org/10.1166/ibn.2012.1367

49. Issa JP, Nascimento C, lyomasa MM, Siéssere S, Regalo SC, Defino HL, et al. Bone healing process in critical-sized defects by rhBMP-2 using poloxamer gel and collagen sponge as carriers. Micron. 2008;39(1):17-24. https://doi.org/10.1016/i.micron.2007.08.008 